\title{
Ortner's syndrome due to large bilharzial pulmonary artery aneurysm
}

\author{
Mahmoud Abdelnabi ${ }^{1}$, Nouran Eshak ${ }^{2}$, Yehia Saleh ${ }^{2}$, Ibrahem Attia ${ }^{1}$, Mahmoud \\ Abdelkader $^{1}$, and Abdallah Almaghraby ${ }^{2}$ \\ ${ }^{1}$ Alexandria University Medical Research Institute \\ ${ }^{2}$ Alexandria University Faculty of Medicine
}

April 28, 2020

\begin{abstract}
Ortner's syndrome is a really rare complication that manifest by hoarseness of voice. It is usually due to left recurrent laryngeal nerve compression. Cardiac causes of Ortner's syndrome are rarely encountered and it is usually due to left atrial enlargement as a complication of valvular lesions affecting mitral valve but other rare causes include ascending aortic aneurysm or pulmonary artery aneurysm. Hereby, we present the 3rd case in the literature to report Ortner's syndrome due to pulmonary artery aneurysm in a 38-year old female patient with previous history of bilharziasis.
\end{abstract}

\section{Case Presentation:}

A 38-year-old woman with a history of bilharziasis presented with progressive dyspnea over the past 5 years and a 1-year-history of horsiness of voice. Chest X-ray showed enlarged cardiac shadow with a dilated pulmonary trunk (Figure 1, Panel A). Echocardiography revealed dilated pulmonary artery and branches and moderate pulmonary regurgitation (Figure 1, Panel B, C) (Video 1,2). Computed Tomography (CT) of Chest and Pulmonary Angiography showed dilatation of the main pulmonary and both pulmonary artery branches. (Figure 1, Panel D, E). Laryngoscopic examination revealed left vocal cord paralysis with immobile vocal cord in paramedian position. She was counselled about surgical decompression but refused surgery. Ortner's syndrome is due to left recurrent laryngeal nerve compression, commonly caused by left atrial enlargement due to mitral stenosis, other rare cardiac causes include thoracic aortic aneurysm, aberrant subclavian artery and pulmonary artery aneurysm. Echocardiography may not satisfactory for a definitive diagnosis. CT is crucial for differential diagnosis of compression causes. Nerve decompression may reverse hoarseness if the duration is not long and the nerve is not permanently damaged. However, ischemic nerve injuries are usually irreversible. 


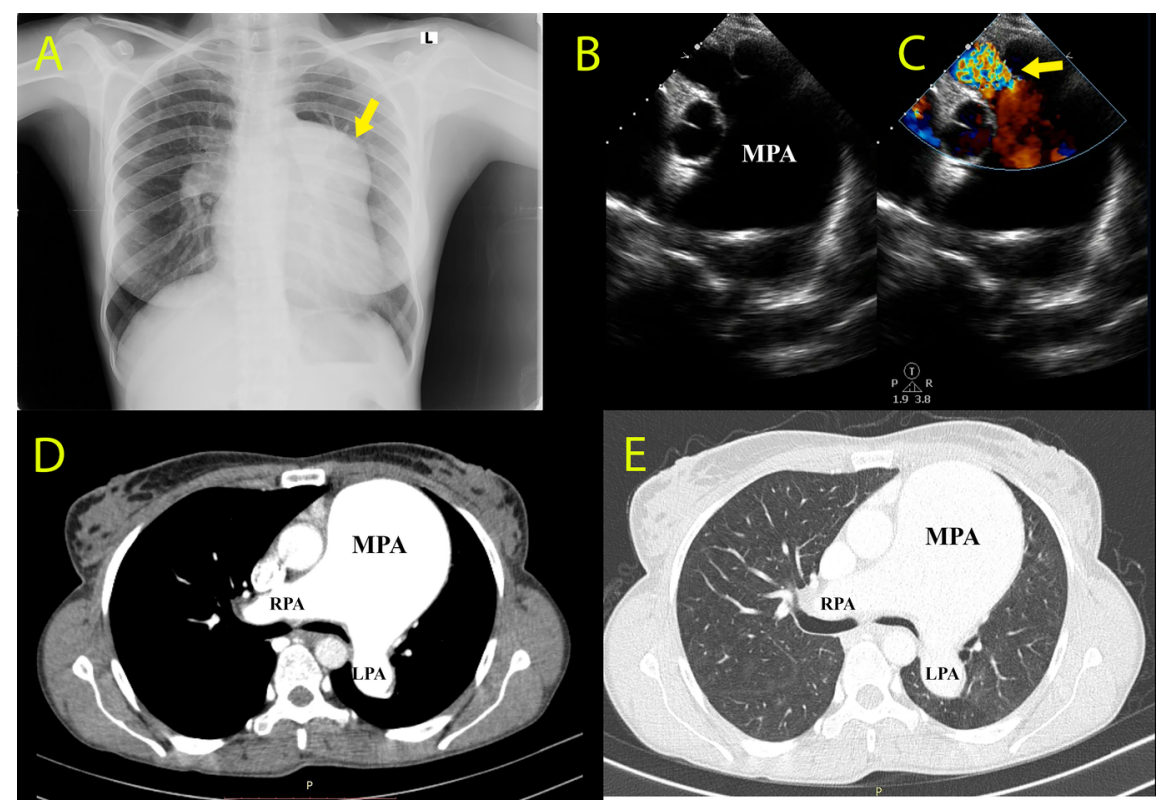

